\title{
Promotion of gastrointestinal endoscopy in Sub-Saharan Africa: What is needed, and how can ESGE and WEO help?
}

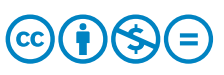

\author{
Authors \\ Purnima Bhat ${ }^{1}$, Cesare Hassan², Hailemichael Desalegn ${ }^{3}$, Lars Aabakken ${ }^{4}$
}

Institutions

1 ANU Medical School/Canberra Hospital Gastroenterology, Garran, Australia. Committee member and Research Lead of Activities to Reach Africa - World Endoscopy Organization

2 Gastroenterology Unit, Nuovo Regina Margherita Hospital, Rome, Italy. Chair of the International Affairs Working Group - European Society of Gastrointestinal Endoscopy; Committee member of Activities to Reach Africa - World Endoscopy Organization

3 Department of Internal Medicine, St. Paul's Hospital Millennium Medical College, Addis Ababa, Ethiopia. Committee member of Activities to Reach Africa World Endoscopy Organization

4 Department of Transplantation Medicine, Faculty of Medicine, Oslo University Hospital - Rikshospitalet, Oslo, Norway. Co-chair of the Committee of Activities to Reach Africa - World Endoscopy Organization
Bibliography

Endosc Int Open 2021; 09: E1001-E1003

DOI 10.1055/a-1495-5215

ISSN 2364-3722

(C) 2021. The Author(s).

This is an open access article published by Thieme under the terms of the Creative Commons Attribution-NonDerivative-NonCommercial License, permitting copying and reproduction so long as the original work is given appropriate credit. Contents may not be used for commercial purposes, or adapted, remixed, transformed or built upon. (https://creativecommons.org/licenses/by-nc-nd/4.0/)

Georg Thieme Verlag KG, Rüdigerstraße 14,

70469 Stuttgart, Germany

Corresponding author

Cesare Hassan, ONRM Hospital - Gastro, Via Morosini 30

Rome 00153, Italy

Fax: +390658446533

cesareh@hotmail.com
The role of gastrointestinal endoscopy in the developed world has seen an amazing expansion that has resulted in it becoming a crucial diagnostic and therapeutic tool to replace radiology, as well as surgery to an increasing extent. Areas of Africa have seen a similar development, but in large areas of Sub-Saharan Africa (SSA), the situation is far from ideal, with very limited access to endoscopic competence. This is related to financial, organizational, and educational factors, which have hampered the development of badly needed endoscopy services. Presence and involvement from relevant industry partners is also variable and often incomplete. General political and logistic challenges specific to each country add to the complexity; however, with a goal-oriented approach, improvement can be achieved within those limitations. Along with the process of improving the overall situation, there is a need to optimize the use of currently available resources. To accomplish this, a comprehensive understanding of local and regional dynamics is essential.

As such, the report from Uganda in this issue of Endoscopy International Open offers potentially important data on the local situation [1]. This study reports findings in dyspeptic patients, based on referrals for endoscopy to their national referral center. The results show a surprisingly high prevalence of what the authors describe as "organic dyspepsia" coupled with a remarkably high incidence of gastroesophageal cancer, especially in those over 50 years of age. Based on these findings, the authors recommend upper gastrointestinal endoscopy in all dyspeptic patients older than 50 years.

Such data are scarce and critical to collect and publish. However, some caution must be used in their interpretation. The organic dyspepsia was mainly caused by "gastritis," as evidenced by endoscopy. This visual diagnosis is a weak indicator of pathophysiology, even of Helicobacter pylori infection [2]. The $>10 \%$ endoscopic prevalence of gastroesophageal cancer is undoubtedly high. However, a selection bias cannot be excluded due to the nature of tertiary centers in the endoscopic facilities studied. Dyspepsia was defined irrespective of alarm symptoms. Thus, most of the dyspeptic patients with cancer were likely to be identified by the alarm symptom rather than by dyspepsia in the first place. Data were collected retrospectively, with accompanying limitations. Finally, the recruitment of patients in this study population is unknown; surely a large proportion of dyspeptic patients were not (and probably should not) be referred for upper endoscopy.

Still, these data are important and the authors should be commended. Where to go from here? 
Generalization of data from one study to the entirety of SSA is difficult. There are significant epidemiological, cultural, and developmental differences that can only be addressed by collection of loco-regional data. Thus, research and data collection in each endoscopy center should be encouraged to the point of being routine. At the same time, the focus should remain broad to gain an understanding of the roles of changing social, economic, and medical factors in disease patterns. WEO and European Society of Gastrointestinal Endoscopy provide a unifying umbrella for collaborative research between endoscopy units nationally and internationally. The World Endoscopy Organization (WEO) research website portal (www.worldendo.org), by facilitating collaboration and providing a forum that serves as a sounding board for research projects, may be a valuable tool in this endeavour. The program facilitates connections between researchers/trainees and mentors, and with each other.

Upper gastrointestinal endoscopy has been widely available in the developed world for several decades, increasing the understanding of dyspepsia and the correlation of findings with patient symptoms, which themselves are closely tied to patient behavior and social structure. This fundamental understanding is still lacking in parts of SSA and more knowledge about societal and behaviour factors specific to the region is critical. Local physicians are the ones best situated to collect and advance this knowledge.

Locoregional research, then, is mandatory. There are many reasons to include research from the outset in these settings. In the first place, measurable outcomes are what drive funding, whether it is from non-governmental organizations or the government. In addition, there is major utility in understanding the case mix to predict departmental needs and to identify systemic shortcomings. In Addis Ababa, this was achieved by implementing custom-designed procedure reporting software, which provided a degree of uniformity in reports and automatically collected parametric data. One early output was from the case mix. It was apparent that the rates of symptomatic dyspepsia were high and associated with gastritis on endoscopy, but the rates of $H$. pylori infection were unknown. This was largely due to the insurmountable cost of rapid tests and histology in a highly endemic population. Thus, the department initiated a research project, funded by the hospital, to develop an inhouse rapid urease test and investigate local antibiotic sensitivities of $H$. pylori.

In another example from the same unit, a comparative study is assessing the efficacy of colonoscopy preparation using locally available laxatives in combination with polyethylene glycolbased prep to improve patient compliance and colonoscopy outcomes. The benefits of this research are self-evident and the results directly applicable. Moreover, the organizing and handling of local research projects inherently improves quality of the service beyond the study itself.

To utilize the data collected and the developing competence, regional quality endoscopic services are required. Beyond the physical centers, this also entails training endoscopists to a high level of procedural competence, but also training nursing staff in procedures, care, and disinfection. Outside experts can help to both set up quality regional hubs for good endoscopic services and also develop train-the-trainer systems to enable these sites to offer regional teaching. Hands-on experience appears critical in this respect. We have established and continue to support several SSA training hubs as the backbone of an active and collaborative endoscopy network. To these were added research training and support, both handson and through the development of a mentorship program [3].

Centralized hubs in developing health care systems offer an excellent opportunity to advance regional endoscopy. However, much of the population of SSA lives in rural regions, where the tyranny of distance and significant costs of travel limit patient mobility. Several solutions have been proposed to address this problem, including mobile gastroenterology units or outreach activities to train local doctors. Remote education programs through locally-initiated online regional seminar series, such as the recently established SSA ECHO from Addis Ababa, have been hugely popular and are highly cost-effective. The recognition that international support brings to these endeavors expands their spheres of influence to include government and industry, which helps to propel the programs further. Furthermore, our societies can enforce such teaching programs with faculty and additional expertise.

Development of endoscopic services requires infrastructure to provide essentials such as endoscopy setup and disinfection facilities, but also hospital infrastructure to handle acquisition of accessories and maintain records and service contracts. These logistical essentials are lacking in most health care centers in the region. Options to address these fundamental factors are limited, but forming a network between centers may serve to provide support and ideas to overcome these limitations. It is hoped that the umbrella of our organizations, meetings, and online forums can provide the networking opportunities these centers require to share knowledge and strategies specific to their region.

Development of quality endoscopy in Africa is essential to deal with regional needs that include, as in developed countries, both emergency services such as variceal banding [4] and diagnostic services such as investigation of dyspepsia. By establishing training hubs in key locations, we hope to be able to assist this development. Training is core, but regional research with robust data collection mechanisms is equally vital, to supplement data like those from Uganda. Involvement of international bodies must be within the context of understanding local needs through hands-on experience. Even more important is the connection with motivated local departmental leadership willing to engage with external experts. The key to rapid progress of endoscopy in Africa lies in successful collaboration.

\section{Competing interests}

The authors declare that they have no conflict of interest. 


\section{References}

[1] Mbiine R, Nakanwagi C, Kituuka O. High rates of gastro-esophageal cancers among patients with dyspepsia undergoing upper $\mathrm{Gl}$ endoscopy in Uganda. Endoscopy International Open 2021; 09: E997E1000

[2] Adu-Aryee NA, Aabakken L, Dedey F et al. Comparison of endoscopic based diagnosis with Helicobacter urease test for Helicobacter pylori infection. BMC Res Notes 2016; 9: 421
[3] Hassan C, Aabakken L, Ebigbo A et al. Partnership with African Countries: European Society of Gastrointestinal Endoscopy (ESGE) - Position Statement. Endosc Int Open 2018; 6: E1247-E1255

[4] Karstensen JG, Ebigbo A, Bhat P et al. Endoscopic treatment of variceal upper gastrointestinal bleeding: European Society of Gastrointestinal Endoscopy (ESGE) Cascade Guideline. Endosc Int Open 2020; 8: E990-E997 